\title{
射频热等离子体制备球形氧化铝粉末的数值模拟及实验研究
}

\author{
陈文波 ${ }^{1,2}$, 陈伦江 ${ }^{2}$, 刘川东 ${ }^{2}$, 程昌明 ${ }^{2}$, 童洪辉 ${ }^{2}$, 朱海龙 ${ }^{3}$
}

(1. 南华大学电气工程学院, 衡阳 421001; 2. 核工业西南物理研究院, 成都 610041 ; 3. 山西大学物理电子工程 学院, 太原 030006)

摘 要: 研究粉末颗粒在热等离子体(ICTP)中的行为可以为射频等离子体制备球形粉末工艺过程的优化提供参考。 首先, 利用 FLUENT 软件对具有不同粒径分布的氧化铝粉末颗粒在射频热等离子体中的运动轨迹及加热历程进行 了数值模拟; 然后, 根据模拟结果所确定的实验参数范围进行了射频热等离子体粉末球化实验, 并将实验测量与 数值模拟的结果相结合，研究了输入功率、送粉速率等参数的改变对具有不同粒径分布的氧化铝粉末球化效果的影 响。研究结果表明: 粒径较小的氧化铝粉末颗粒在飞行过程中可以从等离子体内吸收更多的热量, 因此能够被充分 加热至完全熔化; 增加系统输入功率、降低送粉速率均能提高单位质量的颗粒从等离子体中获得的能量, 从而在一 定程度上提升氧化铝粉末的球化率。

关 键 词: 射频热等离子体; 氧化铝粉末; 运动轨迹; 加热历程; 数值模拟

中图分类号: TB43 文献标识码: A

\section{Preparation of Spherical Alumina Powder by RF Thermal Plasma: Numerical Simulation and Experimentation}

\author{
CHEN Wen-Bo ${ }^{1,2}$, CHEN Lun-Jiang ${ }^{2}$, LIU Chuan-Dong ${ }^{2}$, CHENG Chang-Ming ${ }^{2}$, \\ TONG Hong-Hui ${ }^{2}$, ZHU Hai-Long ${ }^{3}$
}

(1. College of Electrical Engineering, University of south China, Hengyang 421001, China; 2. Southwestern Institute of Physics, Chengdu 610041, China; 3. College of Physics and Electronics Engineering, Shanxi University, Taiyuan 030006, China)

\begin{abstract}
Behavior of alumina powder particles in inductively coupled thermal plasma (ICTP) can provide theoretical reference and guidelines for improving preparation process of plasma spheroidization. In this study, the motion trajectories and heating process of alumina powder particles in ICTP were investigated by means of numerical simulation with FLUENT software. Then the plasma spheroidization experiment was carried out on the basis of simulation results, and the effect of input power, powder feeder rate and particle size distribution on alumina powder spheroidization were studied by combination of experimental and the theoretical analyses. The results show that the small particles absorb enough heat from thermal plasma and therefore be heated to completely melt. Furthermore, the particle can get more energy from plasma while the input power of system is increased or the powder feeder rate is decreased, which improve the spheroidization effect of alumina powder particles.
\end{abstract}

Key words: ICTP; alumina particles; trajectory of particles; heating history; numerical simulation

收稿日期：2017-05-31; 收到修改稿日期：2017-09-06

基金项目：国家自然科学基金(11535003, 11675074); 湖南省教育厅项目(15C1174)

National Natural Science Foundation of China(11535003, 11675074); Project of the Education Department of Human Province (15C1174)

作者简介：陈文波(1983-)，男，博士研究生，讲师. E-mail: snipers2004@163.com

通讯作者：童洪辉, 研究员. E-mail: tonghh@swip.com 
球形氧化铝粉末材料既具有耐高温、抗腐蚀、 耐磨损等优良性能, 还具有流动性好, 振实密度和 松装密度高, 应力应变均匀等常规粉体所不具备的 优点, 在航空航天、机械制造、化学化工等领域有 着广阔的应用前景 ${ }^{[1-2]}$ 。因此, 研究球形氧化铝粉末 的制备技术具有十分重要的现实意义。

射频热等离子体粉末球化技术是一种新型的粉 末材料处理技术。与传统粉末球化方法相比, 射频 热等离子体法工艺简单, 制备的粉末产品球形度 高、表面光洁度好、粉末纯度及松散度也更高, 在 高品质粉末，尤其是难熔球形粉末的制备上更具优 势, 但同时也存在着球化率不高, 均匀性差等缺点, 制约了该技术的工业化应用 ${ }^{[3-6]}$ 。在等离子体粉末球 化实验过程中, 前驱体粒径分布、系统输入功率及 送粉速率等工艺参数是影响最终球化效果的重要因 素。这些参数若仅通过实验方式来进行探索, 不仅 成本高、耗时长, 而且未必找出最优的结果。因此, 结合实验数据进行数值模拟成为研究热等离子体粉 末球化过程最经济有效的手段 ${ }^{[7]}$ 。

目前已有多个研究组采用数值模拟的方式研究 颗粒在等离子体中的行为 ${ }^{[8-18]}$ 。比如, Hossain 等 ${ }^{[11-12]}$ 建立了射频等离子体炬的二维模型, 用数值研究了 等离子体与单个颗粒间的相互耦合效应。Shigeta 研 究团队 ${ }^{[13]}$ 在考虑颗粒受热蒸发对气相场反影响的 前提下, 对颗粒在等离子体中的受力与传热规律进 行了探索。Boulo 小组 ${ }^{[14]}$ 主要研究了氧化铝粉末粒 径在不同工作气体中的变化历程。Colombo 小组 ${ }^{[15]}$ 则分析了射频等离子体炬工作参数的变化对不同性 质粉末颗粒运动轨迹及加热过程的影响效应。但是, 上述研究大多集中于对单个颗粒或单一粒径颗粒束 受力及受热行为的数值模拟 ${ }^{[16-18]}$, 而与具有不同粒 径分布的颗粒束在热等离子体中受力及传热规律相 关的研究却很少。此外, 在实际球化实验过程中, 由 于所采用的工作气体、气流量大小、输入功率及球 化装置尺寸等参数均不相同, 上述研究成果可供借 鉴, 但并不能直接运用到具体的球形粉末制备工艺 中去。

本工作将以核工业西南物理研究院自行研制的 射频等离子体球化装置为研究对象, 在考虑颗粒与 等离子体相互作用的基础上, 利用数值模拟方式考 查输入功率、送粉速率的改变对不同粒径分布的氧 化铝粉末颗粒在热等离子体中的运动轨迹及加热历 程的影响效应, 并结合数值模拟所确定的实验参数 范围进行粉末球化实验。

\section{1 数值模拟方法及实验过程}

射频等离子体球化实验系统如图 1(a)所示，它 主要包括射频电源(工作频率为 $3.0 \mathrm{MHz}$ )、送粉装 置、射频等离子体炬、水冷反应室、粉末收集装置 及尾气排放系统。其中，射频等离子体炬是整个系 统的核心装置, 它由粉枪, 内部石英管, 外部约束陶 瓷管及底部的陶瓷管座构成，其结构示意图如图 1(b) 所示，图中的 $Q_{0} 、 Q_{1}$ 和 $Q_{2}$ 分别为粉末携带气、反 应气及冷却气的气体流量。本文将以图 1 给出的装 置结构为基础进行数值模拟。

\section{1 控制方程组}

假设射频等离子体球化装置内的热等离子体处 于局域热力学平衡(LTE)态, 且等离子体为光学薄 (即认为辐射热损耗仅仅是温度的函数), 则在定常 和层流流动的条件下，描述热等离子体物理行为的 控制方程组可以写为 ${ }^{[23]}$ :

质量守恒方程:

$$
\nabla \cdot(\rho \overline{\boldsymbol{v}})=0
$$

动量守恒方程:

$$
\frac{\partial(\rho \overline{\boldsymbol{v}})}{\partial t}+\nabla \cdot(\rho \overline{\boldsymbol{v}} \overline{\boldsymbol{v}})=-\nabla p+\nabla \cdot \overline{\bar{\tau}}+\rho g+\overline{\boldsymbol{F}}_{\mathrm{L}}
$$

能量守恒方程:

$$
\frac{\partial(\rho h)}{\partial t}+\nabla \cdot(\rho \overline{\boldsymbol{v}} h)=-\nabla \cdot\left(\frac{k_{\mathrm{p}}}{C_{\mathrm{p}}} \nabla h\right)+P_{\mathrm{J}}-Q_{\mathrm{R}}
$$

(a)

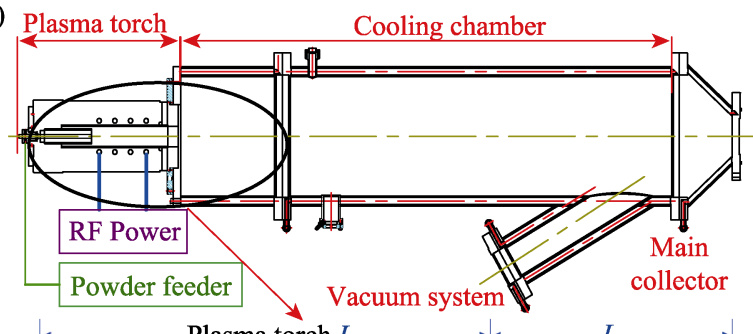

(b)

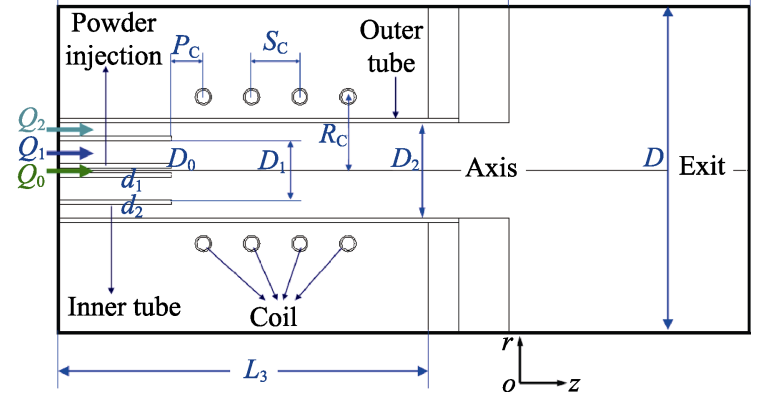

图 1 射频感应等离子体球化系统(a)和等离子体炬装置(b) 结构示意图

Fig. 1 Schematic diagram of inductively coupled plasma spheroidization system (a) and plasma torch structure (b) 
磁矢量势方程:

$$
\nabla^{2} \overline{\boldsymbol{A}}-i \mu_{0} \sigma \omega \overline{\boldsymbol{A}}+\mu_{0} J_{\mathrm{c}}=0
$$

洛仑兹力及焦耳热表达式:

$$
\overline{\boldsymbol{F}}_{\mathrm{L}}=\frac{1}{2} \operatorname{Re}\left(\overline{\boldsymbol{J}} \times \overline{\boldsymbol{B}}^{*}\right), P_{\mathrm{J}}=\frac{1}{2} \operatorname{Re}\left(\overline{\boldsymbol{J}} \cdot \overline{\boldsymbol{E}}^{*}\right)
$$

上述方程组中的 $\overline{\bar{\tau}}=\mu\left[\left(\nabla \overline{\boldsymbol{v}}+\nabla \overline{\boldsymbol{v}}^{T}\right)-\frac{2}{3} \nabla \cdot \overline{\boldsymbol{v}} I\right]$ 为应力张量, $p$ 为压力, $v$ 为速度, $\rho 、 \mu 、 \lambda$ 和 $C_{\mathrm{p}}$ 分别 为等离子体密度, 粘滞系数, 热导率以及常压下的 比热容, $h$ 为等离子体焓值, $Q_{\mathrm{R}}$ 为辐射热损耗; $E$ 和 $B$ 分别为空间中的电场及磁场, $J_{\mathrm{c}}$ 为线圈内的射频电 流密度。

\section{2 粉末颗粒运动及受热模型}

假设氧化铝粉末颗粒在运动过程中只受自身重 力、等离子体流体阻力及热泳力作用的影响, 并忽 略因颗粒蒸发作用而对等离子体的输运特性所造成 的影响, 则颗粒在热等离子体内运动时的受力平衡 方程可表述为:

$$
\frac{\mathrm{d} U_{\mathrm{p}}}{\mathrm{d} t}=\left(\frac{3 \rho C_{\mathrm{D}}}{4 d_{\mathrm{P}} \rho_{\mathrm{p}}}\right)\left(U-U_{\mathrm{p}}\right)\left|U-U_{\mathrm{p}}\right|+g-F_{T}
$$

上式中的 $\rho$ 和 $U$ 分别为等离子体密度和等离子体速 度, $d_{\mathrm{p}}$ 及 $U_{\mathrm{p}}$ 分别为颗粒粒径及颗粒的运动速度; $C_{\mathrm{D}}$ 为阻力系数, 其表达式参见文献[12], $g$ 为重力加速 度, $F_{T}=\left(\frac{36 \mu^{2}}{\rho d_{\mathrm{p}}^{2} \rho_{\mathrm{p}}}\right) B \nabla \ln T$ 为热泳力。

假设颗粒内部温度分布均匀, 则可以通过求解 下述能量平衡方程来获得颗粒温度的变化历程:

$$
C_{\mathrm{p}} m_{\mathrm{p}} \frac{\mathrm{d} T_{\mathrm{p}}}{\mathrm{d} t}=\alpha \pi d_{\mathrm{p}}^{2} \rho C_{\mathrm{D}}\left(T-T_{\mathrm{p}}\right)-\varepsilon \sigma_{\mathrm{SB}} \pi d_{\mathrm{p}}^{2} T_{\mathrm{p}}^{4}
$$

在(7)式中, $C_{\mathrm{p}}$ 表示颗粒的比热。 $\alpha$ 为颗粒表面热 传导系数, $T$ 是等离子体温度, $T_{\mathrm{p}}$ 是颗粒温度, $\varepsilon$ 为颗 粒发射率, $\sigma_{\mathrm{SB}}$ 是 Stefan-Boltzmann 常数。

\section{3 数值求解过程及边界条件}

本文将利用FLUENT 软件来模拟等离子体的流 场分布及氧化铝粉末颗粒在热等离子体中的受力及 受热行为。其中, 磁矢量势方程通过用户自定义标 量(UDS)进行添加, 等离子体的比热容、热导率和粘 度等热力学参数和各控制方程的源项则利用用户自 定义函数(UDF)引入 FLUENT 求解器中。所有的控 制方程均采用二阶迎风格式进行离散, 并采用基于 压力和速度耦合求解器的 SIMPLE 算法求解。热等 离子体中的颗粒运动轨迹及加热历程则通过 DPM 模块来进行求解。由于模型中需要考虑颗粒与外部 流场的相互作用，因此选择耦合求解器。
等离子体炬所有的进气喷口均设置为速度入口 边界, 喷口处粉末携带气、反应气及冷却气的流速 分别为 $11.8 、 0.4$ 和 $1.4 \mathrm{~m} / \mathrm{s}$; 装置内壁面选择无滑移 边界条件; 等离子体炬及反应室以外区域的温度设 置为 $T=300 \mathrm{~K}$; 所有的氧化铝粉末颗粒均以 $U_{\mathrm{p}}=$ $1.9649 \mathrm{~m} / \mathrm{s}$ 的初速度沿轴向注入到等离子体炬中, 颗粒表面的初始温度为 $300 \mathrm{~K}$ 。氧化铝粉末材料的 物理性质见表 1 。

\section{4 射频等离子体球化实验过程}

1)将等离子体炬及反应室内抽成真空；2)通入 工作气体(氩气), 接入高压击穿氩气形成高密度热 等离子体, 并通过调节氩气流量来稳定等离子体电 弧；3)根据数值模拟结果调整系统输入功率和送粉 速率，将具有不同粒径分布的氧化铝前驱体经粉枪 送入等离子体炬内进行多组实验; 4)利用扫描电子 显微镜(SEM)观察从系统底部收集装置中收集到的 球形氧化铝粉末颗粒的表观形貌，利用激光衍射粒 度分析仪分析表征颗粒的粒度分布。

\section{2 结果与讨论}

\section{1 数值模拟结果}

首先研究前驱体粉末粒径分布的变化对氧化铝 颗粒在等离子体中受力及受热过程的影响。当送粉 速率为 $36 \mathrm{~g} / \mathrm{min}$, 输入功率 $P=63 \mathrm{~kW}$ 时, 计算得到 的粒径分布分别为 $20 \sim 30 \mu \mathrm{m}$ 及 $40 \sim 50 \mu \mathrm{m}$ 的两组颗 粒在等离子体中的运动轨迹及颗粒平均温度的变化 历程分别如图 2 和图 3 所示。

从图 2 中可以看出, 粒径分布为 $20 \sim 30 \mu \mathrm{m}$ 的颗 粒由于质量较轻, 受等离子体球化装置内流场作用 的影响较大, 可以在等离子体高温区内停留较长的 时间，因此能够从等离子体中吸收足够多的热量, 从而达到更好的球化效果。从图 3 中颗粒束平均温 度沿轴向的变化情况来看, 该组颗粒平均温度达到 了材料沸点, 说明所有颗粒均处于强烈蒸发的状态; 粒径分布为 40 50 $\mu \mathrm{m}$ 粉末颗粒主要受重力场的作 用，其运动轨迹更为集中，大部分颗粒会在较短的 时间内沿装置中心穿过高温弧区，从等离子体中吸 收到的热量相对较少。图 3 中计算结果还表明: 在

表 1 氧化铝粉末材料物理性质

Table 1 Physical properties for alumina powders

\begin{tabular}{cccccc}
\hline $\begin{array}{c}\text { Density } \\
/\left(\mathrm{kg} \cdot \mathrm{m}^{-3}\right)\end{array}$ & $\begin{array}{c}\text { Specific } \\
\text { heat } \\
/\left(\mathrm{J} \cdot \mathrm{kg}^{-1}\right. \\
\left.\mathrm{K}^{-1}\right)\end{array}$ & $\begin{array}{c}\text { Melting } \\
\text { point } \\
/ \mathrm{K}\end{array}$ & $\begin{array}{c}\text { Fusion } \\
\text { enthalpy } \\
/\left(\mathrm{J} \cdot \mathrm{kg}^{-1}\right)\end{array}$ & $\begin{array}{c}\text { Boiling } \\
\text { point } \\
/ \mathrm{K}\end{array}$ & $\begin{array}{c}\text { Vaporiza- } \\
\text { tion } \\
\text { enthalpy } \\
/\left(\mathrm{J} \cdot \mathrm{kg}^{-1}\right)\end{array}$ \\
\hline 3900 & 1020 & 2326 & $1.07 \times 10^{6}$ & 3800 & $2.466 \times 10^{7}$ \\
\hline
\end{tabular}




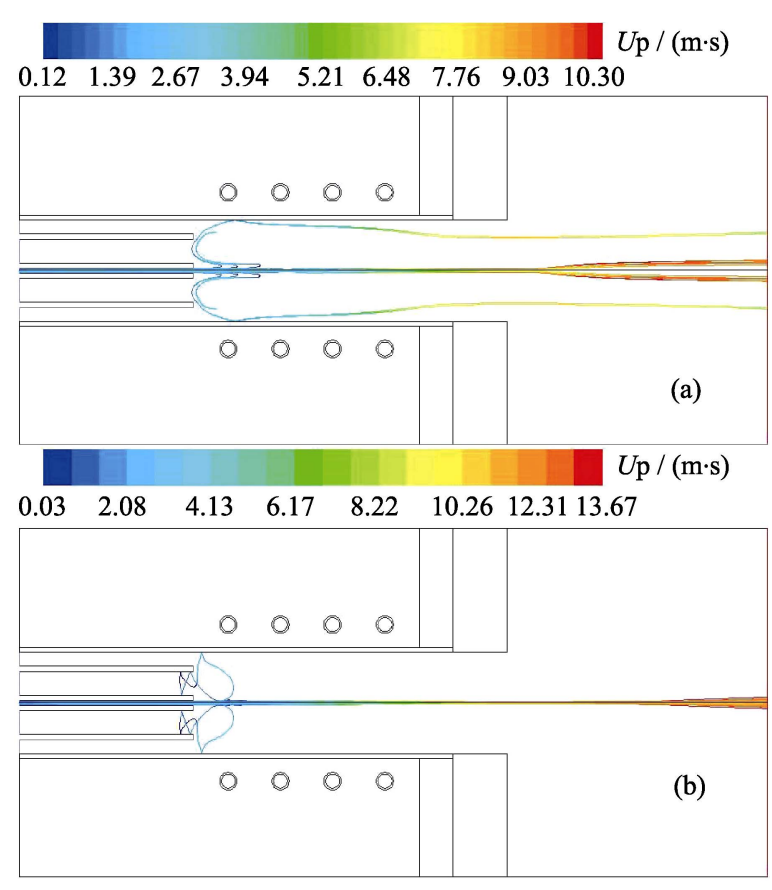

图 2 不同粒径分布的氧化铝颗粒在热等离子体中的运动轨迹 Fig. 2 Trajectory of alumina particles with different particle sizes

(a) $D_{\mathrm{p}}=20-30 \mu \mathrm{m}$; (b) $D_{\mathrm{p}}=40-50 \mu \mathrm{m}$

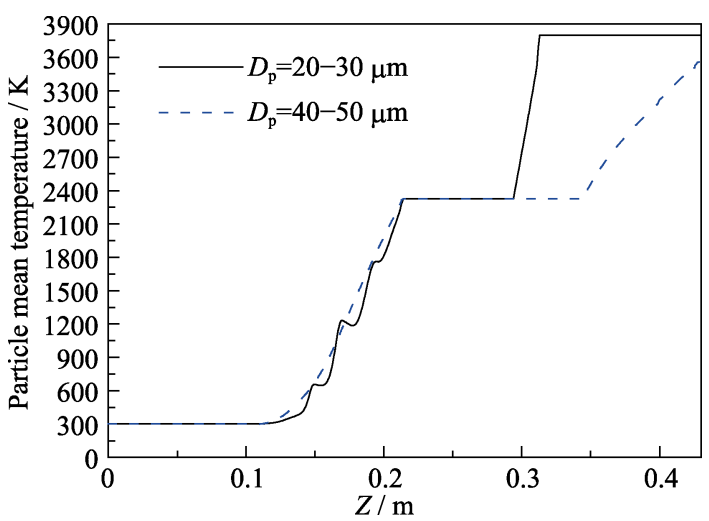

图 3 不同粒径分布的氧化铝颗粒温度

Fig. 3 Particle mean temperature of alumina particles with different particle size distribution

相同的条件下, 粒径为 $40 \sim 50 \mu \mathrm{m}$ 的颗粒束平均温 度仅为 $3565 \mathrm{~K}$, 这说明该组颗粒可以被加热至完全 熔化，但不会蒸发。

当其它工艺参数均不改变, 而等离子体球化装 置的输入功率在 $56 \sim 70 \mathrm{~kW}$ 范围内变化时, 计算得 到的粒径为 20 30 $\mu \mathrm{m}$ 的 $\mathrm{Al}_{2} \mathrm{O}_{3}$ 粉末颗粒束的加热历 程、运动轨迹, 以及装置内等离子体温度分布, 如图 4 6 所示。计算结果表明, 在 $\mathrm{Al}_{2} \mathrm{O}_{3}$ 颗粒束被注入到球 化装置后，总会有部分粒径较小的颗粒在流场作用 下偏离轴线，向等离子体炬陶瓷管的内壁面运动。 当输入功率为 $P=56 \mathrm{~kW}$ 时, 球化装置内的等离子体 温度较低, 无法将偏离轴线运动的颗粒加热至完全

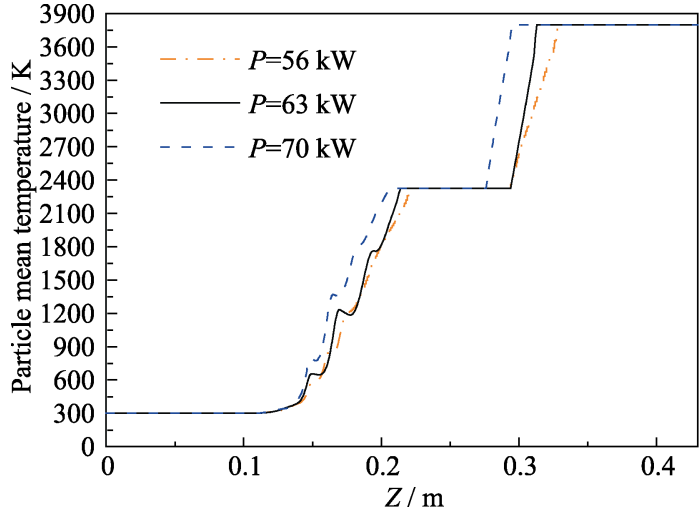

图 4 不同输入功率下, 氧化铝颗粒温度变化历程

Fig. 4 Particle mean temperature of alumina particles with different input powers

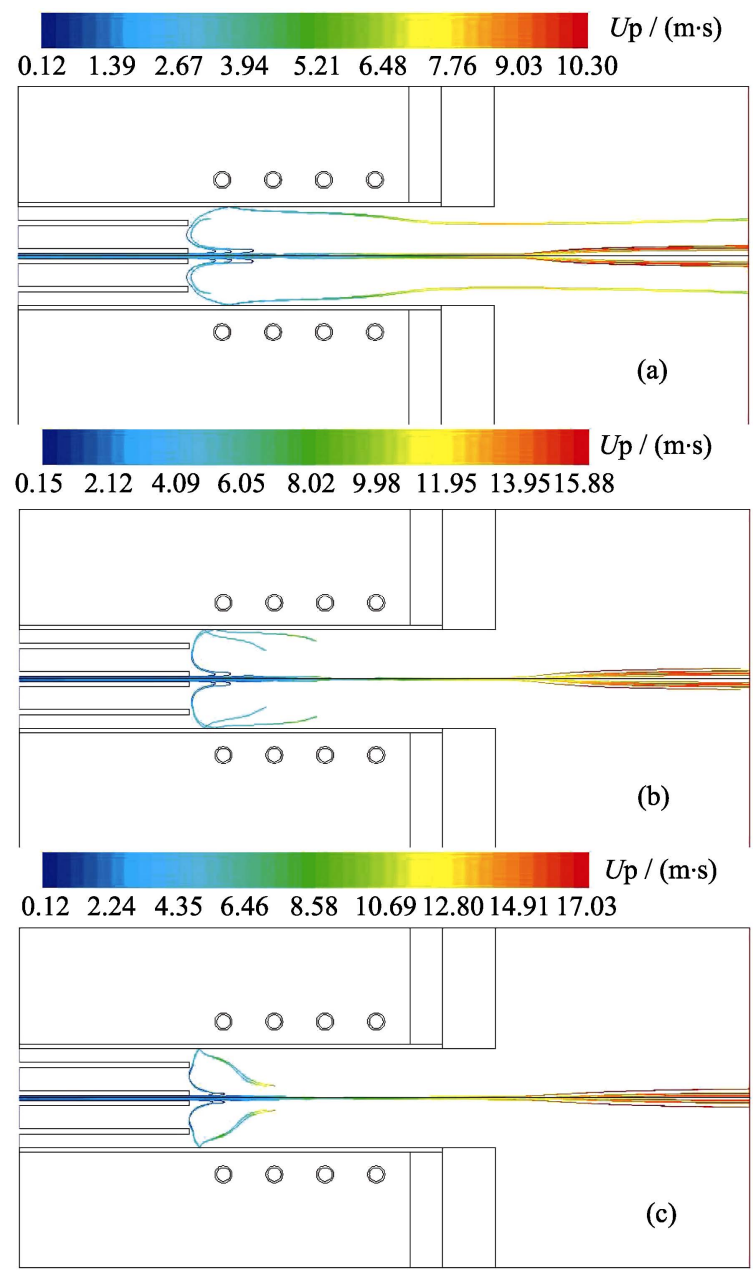

图 5 不同输入功率下, 氧化铝颗粒在热等离子体中的运动 轨迹

Fig. 5 Trajectory of alumina particles with different input powers

(a) $P=56 \mathrm{~kW}$; (b) $P=63 \mathrm{~kW}$; (c) $P=70 \mathrm{~kW}$

蒸发，因此，这部分颗粒将沿陶瓷管内壁面向反应 室出口处继续运动, 并反过来影响到装置内部的温 度分布，使陶瓷管内壁面附近的等离子体温度明显 下降。而当输入功率增加至 $63 \mathrm{~kW}$ 时, 球化装置内 

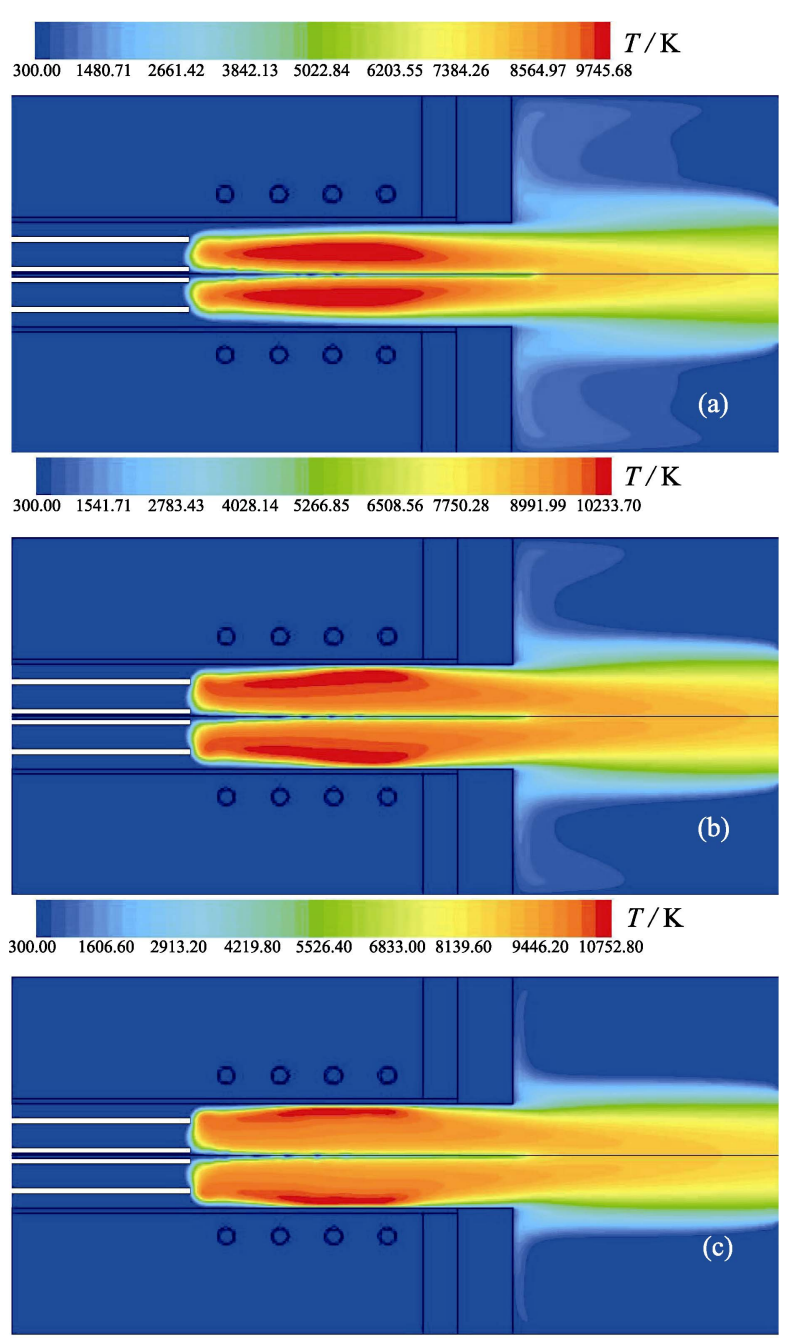

图 6 不同输入功率下, 等离子体炬内温度空间分布图

Fig. 6 Spatial distribution of temperature fields with different input powers

(a) $P=56 \mathrm{~kW}$; (b) $P=63 \mathrm{~kW}$; (c) $P=70 \mathrm{~kW}$

的等离子体最高温度可达 $10000 \mathrm{~K}$ 以上, 且高温区 域靠近陶瓷外管内壁, 这种温度分布可将偏离轴线 运动的小粒径颗粒加热至完全蒸发。因此, 输入功 率越高, 颗粒注入对等离子体温度场所产生的影响 越小，同时颗粒从等离子体中吸收的热量也越多, 这对于粉末球化率的提高是有益的。

送粉速率是优化等离子体球化处理过程中另一 个重要的工艺参数, 在装置输入功率不变 $(P=63 \mathrm{~kW})$ 的情况下, 送粉速率的变化(6.0 96 $\mathrm{g} / \mathrm{min})$ 对粒径为 $20 \sim 30 \mu \mathrm{m}$ 的 $\mathrm{Al}_{2} \mathrm{O}_{3}$ 颗粒束受力及受热行为的影响, 如图 7 所示。不同送粉速率下, 从装置内等离子体 温度分布的模拟结果可以看出, 在 $\mathrm{Al}_{2} \mathrm{O}_{3}$ 颗粒束注 入等离子体后, 颗粒运动轨迹附近的等离子体温度 下降明显，且送粉速率越大，这种冷却效应越强烈。 由图 8 列出的颗粒平均温度的计算结果表明, 当送 粉速率在 $36 \mathrm{~g} / \mathrm{min}$ 以下时, 所有颗粒的温度均能达 到材料沸点; 当送粉速率为 $66 \mathrm{~g} / \mathrm{min}$ 时, 颗粒能够
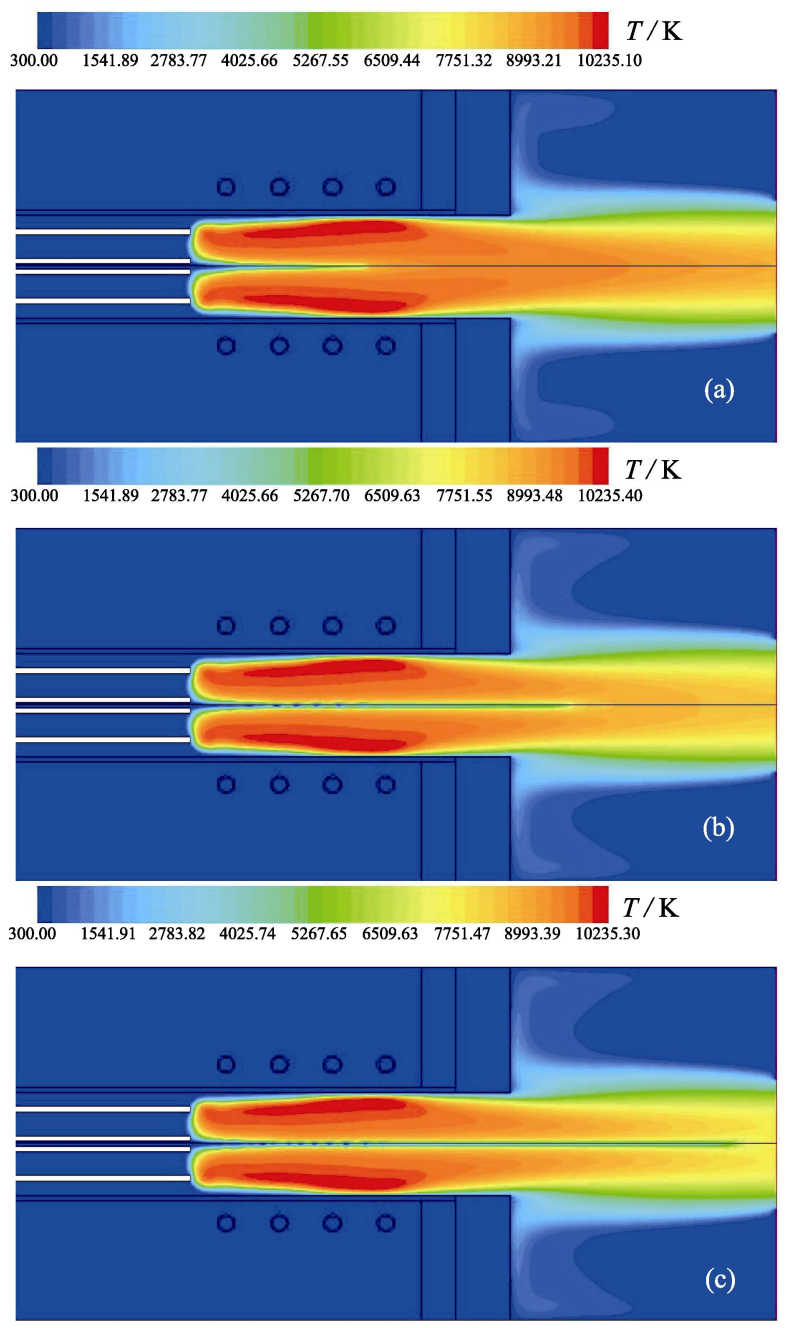

图 7 不同送粉速率下，等离子体炬内温度空间分布图

Fig. 7 Distribution of temperature fields with different powder feed rates

(a) $6.0 \mathrm{~g} / \mathrm{min}$; (b) $66.0 \mathrm{~g} / \mathrm{min}$; (c) $96.0 \mathrm{~g} / \mathrm{min}$

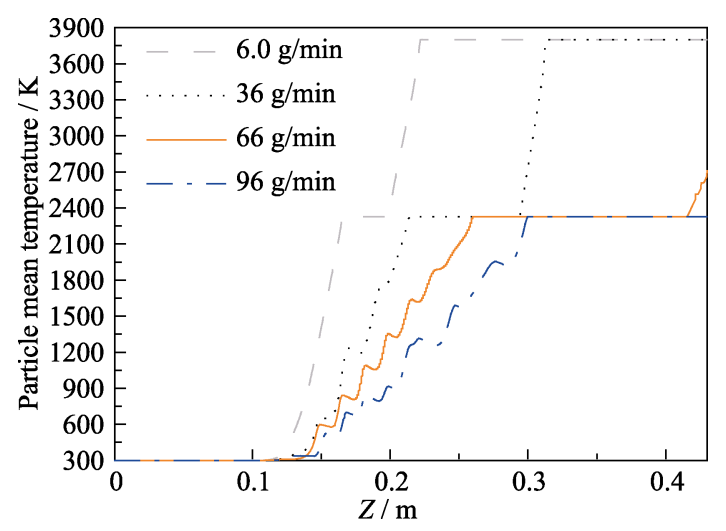

图 8 不同送粉速率时氧化铝颗粒温度变化历程

Fig. 8 Particle mean temperature of alumina particles with different powder feed rates

被加热至完全熔融，但并未出现蒸发的现象; 而当 送粉速率为 $96 \mathrm{~g} / \mathrm{min}$ 时, 由于单位质量的颗粒在反 应器中获得的能量过小, 颗粒束平均温度仅等于材 料熔点，说明仅有部分颗粒能够达到熔化状态。 


\section{2 等离子体球化实验结果}

数值模拟结果表明, 当系统的输入功率在 $56 \mathrm{~kW}$ 时, 送粉速率在 $66 \mathrm{~g} / \mathrm{min}$ 以下即可使平均粒 径在 $50 \mu \mathrm{m}$ 以下的氧化铝颗粒完全熔化。根据这一 结果, 本研究确定的球化实验参数范围如表 2 所示。

从图 9 给出的 1 \#粉末球化后的 SEM 照片可以 看出, 平均粒径为 $45 \mu \mathrm{m}$ 的颗粒球形度良好, 但表 面斑驳，出现明显裂痕，这说明 1 \#粉末颗粒仅有部 分熔融, 在飞出等离子体后的冷却过程中, 内外层 温度不均使得颗粒表面在应力作用下形成裂痕。

图 10 给出了以不同的送粉速率对 $2 \mathrm{AAl}_{2} \mathrm{O}_{3}$ 颗粒 进行球化处理前后的 SEM 照片。从图 10(b)可以看

表 2 射频感应等离子体炬尺寸

Table 2 Dimensions of RF inductively coupled plasma torch

\begin{tabular}{ccc}
\hline Parameter & 1\# Particle & 2\# Particle \\
\hline Particle mean diameter $/ \mu \mathrm{m}$ & 45 & 25 \\
Plasma power $/ \mathrm{kW}$ & 63.75 & 56.00 \\
Carry gas flow rate $/\left(\mathrm{L} \cdot \mathrm{min}^{-1}\right)$ & 5 & 5 \\
Sheath gas flow rate $\left(\mathrm{L} \cdot \mathrm{min}^{-1}\right)$ & 70 & 70 \\
Center gas flow rate $/\left(\mathrm{L} \cdot \mathrm{min}^{-1}\right)$ & 34.5 & 34.5 \\
Powder feed rate $/\left(\mathrm{g} \cdot \mathrm{min}^{-1}\right)$ & 35.0 & $35.0 \sim 65.7$ \\
Chamber pressure $/ \mathrm{kPa}$ & 40 & 40 \\
\hline
\end{tabular}

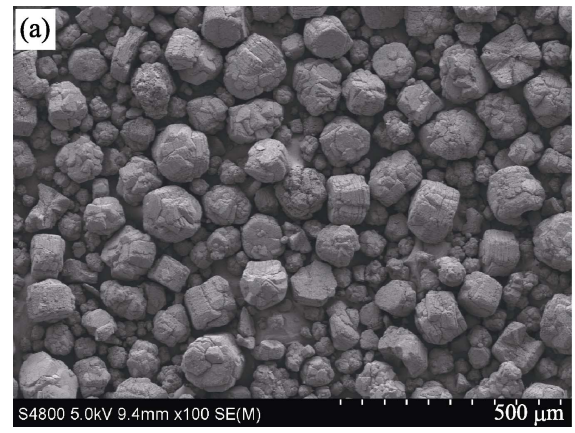

出, 1 \#粉末颗粒在以 $35 \mathrm{~g} / \mathrm{min}$ 的送粉速率进行球化 处理后，粉末颗粒的球形度极高，表面较光滑，且 球化率在 $95 \%$ 以上。这说明尽管 $1 \#$ 粉末是在较低的 输入功率下进行球化实验, 但由于粉末的粒径较小, 球化效果却更好。而图 10(c)则表明，当送粉速率提 高至 $65.7 \mathrm{~g} / \mathrm{min}$ 时, 球化率降至 $60 \%$ 以下, 这与数 值模拟所得出的结论一致。因此, 对于平均粒径在 $50 \mu \mathrm{m}$ 以下的氧化铝原粉颗粒, 兼顾粉末的球化 率与产出率, 其送粉速率应控制在为 $40 \mathrm{~g} / \mathrm{min}$ 左 右为宜。

\section{3 结论}

采用实验与数值模拟相结合的方法, 对射频热 等离子体球化氧化铝粉末的过程进行了研究, 得到 的主要结论如下:

1) 当粉末颗粒粒径较小时, 主要受到流场作 用力的影响，颗粒在等离子体中停留的时间更长， 能够从等离子体中吸收的热量更多，更易被加热至 完全熔化，从而达到较好的球化效果;

2) 提高输入功率会在一定程度上提升等离子体 温度，这将使注入装置内的颗粒温度更快地达到材 料熔点, 从而提高粉末球化率;

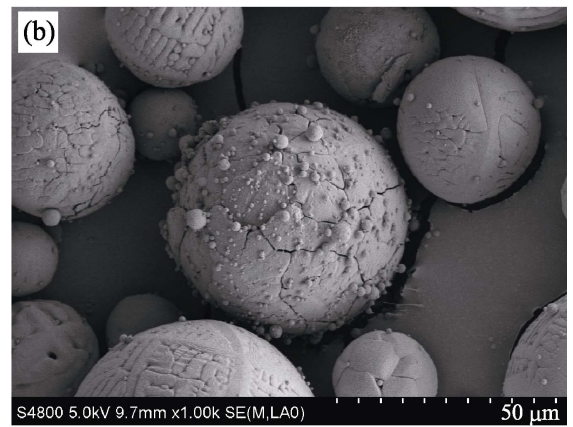

图 91 \#氧化铝颗粒的 SEM 照片

Fig. 9 SEM images of 1 \# alumina particles

(a) Precursor; (b) After being spheroidization
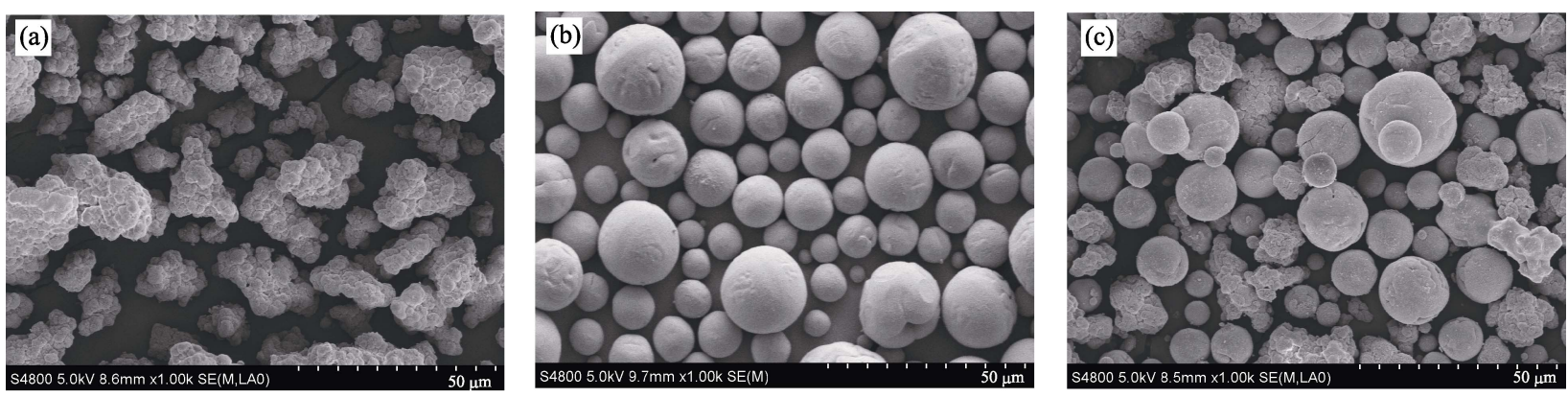

图 102 2\#氧化铝颗粒的 SEM 照片

Fig. 10 SEM images of 2\# alumina particles

(a) Precursor; (b) Powder feed rate at $35 \mathrm{~g} / \mathrm{min}$; (c) Powder feed rate at $65.7 \mathrm{~g} / \mathrm{min}$ 
3) 过快的送粉速率会对装置内的等离子体温度 分布产生较强的冷却效应, 并导致氧化铝粉末的球 化率降低。因此在实验过程中, 可以通过合理地调整 送粉速率来获得最佳球化效果。

\section{参考文献:}

[1] HE D L, MICHAEL B, MAXIME G, et al. Diameter and length dependent self organizations of multi-walled carbon nanotubes on spherical alumina micro-particles. Carbon, 2010, 48(4): 244-248.

[2] LI H T, LIAO Q L, WANG F. Preparation of millimeter-scale alumina hollow spheres by oill-in-water-in-oil2 emulsions. Journal of Inorganic Materials, 2016, 31(3): 329-336.

[3] ZHU H L, TONG H H, YANG F Z, et al. A comparative study on RF thermal plasma spheroidiation for two alumina ceramic powder. Advanced Materials Research, 2014, 58(10): 221-225.

[4] GU Z T, YE G Y, JIN Y P. Chemical compositions of spherical titanium powders prepared by RF induction plasma. High Power Laser and Particle Beams, 2012, 24(7): 1409-1412.

[5] LEE W Y, CHOIC S S, OH S M, et al. Preparation of spherical hollow alumina particles by thermal plasma. Thin Solid Films, 2013, 529(1): 394-397.

[6] KAROLY Z, SZEPVOLGYI J. Hollow alumina microspheres prepared by RF thermal plasma. Powder Technol., 2003, 132(1): 211215.

[7] BOULOS M I. The role of transport phenomena and modeling in the development of thermal plasma technology. Plasma Chem. Plasma Process, 2016, 36: 3-28.

[8] FROLOV V, IVANOV D, SHIBAEV M. Mathematical modeling of plasma technology for $\mathrm{TiO}_{2}$ fine powder production. Digest Journal of Nanomaterials and Biostructures, 2014, 9(3): 12331240.

[9] COLOMBo V, GHEDINI E, GHERARDi M, et al. A two- dimensional nodal model with turbulent effects for the synthesis of Si nano-particles by inductively coupled thermal plasmas. Plasma Sources Science \& Technology, 2012, 21(2): 025001.

[10] RAMASAMY R, SELVARAJANA V. Numerical studies on velocity, temperature history and heat transfer to the particles injected into the argon plasma. The European Physical Journal D, 2001, 15: 229-235.

[11] HOSSAIN M M, YAO Y, WATANABE T. A numerical analysis of plasma-particle heat exchange during in-flight treatment of granulated powders by argon-oxygen induction thermal plasmas. Thin Solid Films, 2008, 516: 6634-6639.

[12] HOSSAIN M M, ALAM M R. Thermal treatment of $\mathrm{Al}_{2} \mathrm{O}_{3}, \mathrm{MgO}$, and $\mathrm{CeO}_{2}$ granulated powders by induction thermal plasma: a numerical approach. Japanese Journal of Applied Physics, 2013, 52(1): 219-233.

[13] SHIGETA M, SATO T, NISHIYAMA H. Computational simulation of a particle-laden RF inductively coupled plasma with seeded potassium: a numerical approach. International Journal of Heat and Mass Transfer, 2004, 47: 707-716.

[14] YE R, ISHIGAKI T, JUREWICZ J, et al. In-flight spheroidization of alumina powders in $\mathrm{Ar}-\mathrm{H}_{2}$ and $\mathrm{Ar}-\mathrm{N}_{2}$ induction plasmas. Plasma Chemistry and Plasma Processing, 2004, 24(4): 555-571.

[15] BERNARDI D, COLOMBO V, GHEDINI E, et al. 3-D numerical simulation of fully-coupled particle heating in ICPTs. The European Physical Journal D, 2004, 28: 423-433.

[16] YE R, LI J G, ISHIGAKI T. Controlled synthesis of alumina nanoparticles using inductively coupled thermal plasma with enhanced quenching. Thin Solid Films, 2007, 515: 4251-4257.

[17] TONG J B, LU X, LIU C C, et al. Numerical simulation and prediction of radio frequency inductively coupled plasma spheroidization. Applied Thermal Engineering, 2016, 100: 1198-1206.

[18] LU X ZHU L P, ZHANG B, et al. Simulation of flow field and particle trajectory of radio frequency inductively coupled plasma spheroidization. Computational Materials Science, 2012, 65: 13-18. 